\title{
Biophysical characteristics of gap junctions in vascular wall cells: implications for vascular biology and disease
}

P.R. Brink ${ }^{1}$,

J. Ricotta ${ }^{2}$ and G.J. Christ Ch, $^{3,4}$

\section{Correspondence \\ P.R. Brink \\ Department of Physiology and \\ Biophysics \\ SUNY at Stony Brook \\ Stony Brook, NY 11794 \\ USA}

Presented at the Meeting

"Gap Junctions in the Nervous and

Cardiovascular Systems: Clinical

Implications", Rio de Janeiro, RJ,

Brazil, June 6-11, 1998.

Research supported by NIH grant GM 55263 .

Received August 13, 1999 Accepted November 23, 1999
Departments of ${ }^{1}$ Physiology and Biophysics, Institute for Molecular Cardiology, and 25urgery, SUNY at Stony Brook, Stony Brook, NY, USA

Departments of ${ }^{3} \mathrm{U}$ rology, and 4 Physiology and Biophysics, Institute for Smooth Muscle Biology, Albert Einstein College of Medicine, Bronx, NY, USA

\section{Abstract}

The role gap junction channels play in the normal and abnormal functioning of the vascular wall is the subject of much research. The biophysical properties of gap junctions are an essential component in understanding how gap junctions function to allow coordinated relaxation and contraction of vascular smooth muscle. This study reviews the properties thus far elucidated and relates those properties to tissue function. We ask how biophysical and structural properties such as gating, permselectivity, subconductive states and channel type (heteromeric $v s$ homotypic $v s$ heterotypic) might affect vascular smooth muscle tone.

\section{Introduction}

In vascular smooth muscle the role that gap junctions play is integral among a number of other processes and parameters influencing tissue function (1-4). The ultimate tissue function, however, is coordinated relaxation and contraction of the many cells within the wall of any particular vessel. Ultimately, the major factors which influence such coordinated tissue behavior are neuronal innervation density, cell to cell communication and cellular excitability (1,5-7). As vascular smooth muscle cells do not tend to generate action potentials, innervation density and gap junction-mediated communication become paramount as potential regulatory sites. The former parameter is the

\section{Key words}

- Vascular smooth muscle

- Gap junctions subject of modeling strategies which rely on gap junction-mediated communication to produce tissue responses to stimuli which mimic reality $(8,9)$. For this reason, the biophysical characteristics of gap junction channels in vascular smooth muscle and associated vascular wall cells, such as endothelial cells, should be elucidated. In particular, for example, are there gap junctional properties which lend themselves to dynamic regulation? How many gap junction channels are needed to allow cells to act syncytially? What types of gap junction channels are present in the vascular wall? Is it possible that hybrid channel types exist in vascular smooth muscle, and if so, would their presence affect tissue function? These issues are considered herein. 


\section{Results and Discussion}

\section{Generic gap junction channel types in vascular tissues}

The subunit protein of any gap junction channel is the connexin and in vascular smooth muscle connexin $43(\mathrm{Cx} 43)$ is the most abundant, followed by connexin 40 (Cx40), connexin45 (Cx45) and possibly connexin37 (Cx37) (1,10-12). Endothelial cells are also able to co-express $\mathrm{Cx} 43, \mathrm{Cx} 40$ and $\mathrm{Cx} 37$, the latter being the most abundant in situ (see 1 for review).

The putative presence of multiple connexin subtypes in a given vascular smooth muscle cell has potentially important physiological implications. For example, there are three types of gap junction channels possible when more than one connexin is being expressed in the same cell. They are as follows: 1) a homotypic gap junction channel composed of 12 identical connexin subunits, 2) a heterotypic gap junction channel where all the connexins of one cell are identical but different from the connexins of the adjacent cell, and 3) the heteromeric gap junction channel where the connexins in any one cell are not identical within a hemichannel. In the case of vascular smooth muscle cells the dominant connexin is $\mathrm{Cx} 43$. Therefore, we will focus our discussion on properties of homotypic gap junction channels composed of $\mathrm{Cx} 43, \mathrm{Cx} 40, \mathrm{Cx} 45$ and $\mathrm{Cx} 37$. Further we will compare homotypic $\mathrm{Cx} 43$ and $\mathrm{Cx} 37$ to the in vitro documentation of $\mathrm{Cx} 43-\mathrm{Cx} 37$ heterotypics and heteromerics. The relevant properties to be described are gating and permselectivity.

\section{Homotypic gap junction membrane voltage dependence}

In general, all mammalian homotypic gap junction channels display symmetric voltage dependence. Because of its ubiquity we will first characterize $\mathrm{Cx} 43$ homotypic gap junc- tion channels and subsequently compare their behavior to $\mathrm{Cx} 37, \mathrm{Cx} 40$ and $\mathrm{Cx} 45$ whenever possible. In this regard, the instantaneous junctional current for $\mathrm{Cx} 43$ is linear over a large voltage range. Transjunctional voltage steps of sufficient amplitude and duration cause junctional currents to decline with time, resulting in a steady-state conductance which is a fraction of the instantaneous conductance. The time course of the voltagedependent behavior is of the order of hundreds of milliseconds to seconds (13). Cx43 shows relatively symmetric voltage dependence but has different voltage sensitivity relative to $\mathrm{Cx} 37$. That is, the point on the $\mathrm{V}_{\mathrm{j}}$ axis where junctional conductance is $50 \%$ of the maximum (i.e., $\mathrm{V}_{\mathrm{o}}$ ) is approximately 25 $30 \mathrm{mV}$ for $\mathrm{Cx} 37$ and $70-85 \mathrm{mV}$ for $\mathrm{Cx} 43$ $(12,14,15)$. Cx40 has a $V_{0}$ in the $40 \mathrm{mV}$ range, while the $\mathrm{V}_{\mathrm{o}}$ for $\mathrm{Cx} 45$ has been reported to be $20 \mathrm{mV}$ (16). Such observations emphasize that, with the possible exception of $\mathrm{Cx} 45$, the voltage sensitivity of connexins in vascular wall cells is not likely to effect dynamic changes in intercellular coupling.

Analysis of the junctional gating over many seconds to minutes has revealed, in the case of $\mathrm{Cx} 43$, another wrinkle to gating, and that is mode shifting. The process has been described in detail (13). Two important features must be considered here. First, the time course to reach steady state was of the order of $60 \mathrm{~s}$ or longer, and second, the mechanism could not be explained as a simple reduction of mean open time or increase in mean closed time for an identical population of channels. Rather, a large portion of the channel population $(90 \%)$ underwent a shift to prolonged silence (prolonged closure) leaving only a small population of functional channels. The process was reversible. Again, this is a voltage-dependent process and the magnitude and duration of the voltage steps $(30-50 \mathrm{mV}$, with durations of many seconds) seem to be outside the physiologically useful range. However, it should be pointed out that if this 
process is modified or driven in the presence of physiologically important ligands/intracellular messengers, such as cAMP or $\mathrm{IP}_{3}$, it could well be a regulatory point with great dynamic range. This latter postulate has yet to be experimentally tested, let alone shown to be functional under physiological conditions. Mode shifting has not been characterized for $\mathrm{Cx} 37$ but has been demonstrated in A7r5 cells which co-express $\mathrm{Cx} 40$ and $\mathrm{Cx} 43$ (He DS, personal communication). Thus, voltage-dependent gating, a common property of all the vertebrate connexins thus far studied, seems to be an unlikely tool for cells to utilize in order to affect dynamic alterations in cell to cell coupling mediated by gap junctions. Whether ligand gating, linked with processes such as mode shifting, might be able to provide dynamic behaviors that are physiologically relevant to the vascular wall cell has yet to be determined.

\section{Homotypic gap junction channel conductance}

The unitary conductance for $\mathrm{Cx} 43$ is 90 $120 \mathrm{pS}(13,17-20)$. Table 1 lists the unitary conductances for all four connexin types found in the vascular system. The different unitary conductance values imply that different permselectivity properties may exist for distinct connexins. What becomes important then, is determining if differential conductance values translate into vessel-specific behaviors of the vascular wall, such as, for example, propagated vasodilation or constriction (21).

\section{Subconductive states: are they physiologically relevant?}

All gap junction channels thus far studied have displayed subconductive states. One common feature is the predominance of subconductive states with large transjunctional voltages, the classic example of this being Cx37 (22) and Cx43 (24). The observation that the permselective properties of the subconductive states are very different from the main state conductance has given rise to much speculation. For example, if the subconductive state is the dominant one in terms of dwell time, then it would necessarily follow that the permeation rates of solutes seeking transit from cell to cell would be quite dependent on the nature of the subconducting state(s). However, before even addressing the permselective properties, the first question answered must be how much of the open time is dominated by subconductive states. If the dwell time in any given subconductance state is significant $(10-20 \%$ or greater?), then the permeation characteristics of the distinct subconducting states become of interest. For Cx43, Christ and Brink (24) have determined that the occupancy time of the subconductive state(s), for a transjunctional voltage of $40 \mathrm{mV}$, is less than $2 \%$ for a channel whose open probability is on the order of 70$90 \%$. In this case, even if the permselectivity of the subconductive state is dramatically different from the main state it would seem hardly relevant to macroscopic tissue behavior with such a low occupancy time. Conversely, if gap junction channels are driven into subconductive states by interaction with ligands, or alterations in second messenger levels, such that for the majority of the open time the conductive and permeation rates are dictated by the subconductive state, then this property of gap junction channels would have very telling affects on tissue function.

Table 1 - Unitary conductance of the four connexin types found in the vascular system.

\begin{tabular}{lcl} 
Connexin & $\begin{array}{c}\text { Unitary conductance } \\
\text { (picoSiemens, pS) }\end{array}$ & Reference \\
\hline Cx37 & $350-400$ & 22,14 \\
Cx40 & 160 & 23 \\
Cx43 & $90-115$ & 13,19 \\
Cx45 & $25-30$ & 16,18
\end{tabular}




\section{Channel permselectivity: its role in tissue physiology}

The homotypic $\mathrm{Cx} 43$ gap junction channel has been studied the most with respect to permselectivity. A recent study by Wang and Veenstra (20) delineated the permeability of Cx43 to monovalent cations. The analysis revealed that homotypic $\mathrm{Cx} 43$ is non-selective, and based on comparison of unitary conductance, follows an Eisenmann series I or II sequence (25), i.e., $\mathrm{Rb}=\mathrm{Cs}>\mathrm{K}>\mathrm{Na}>$ $\mathrm{Li}>\mathrm{TMA}>\mathrm{TEA}$. The anion series also shows a proportionality between mobility and unitary conductance. Hence $\mathrm{Cx} 43$ appears to be very poorly selective and falls into the category of Porin OmpF (26-28) which itself is permeable to both cations and anions. However, it should be noted that the porins have no sequence homology with the connexins. Cx40 follows the same cation sequence, but has a very different anion sequence as that described for $\mathrm{Cx} 43$ and $\mathrm{Cx} 37$. In fact, unitary conductance was slightly decreased with increased ionic mobility, indicating that $\mathrm{Cx} 40$ is much less anion permeable and/or that the cation-anion environment surrounding or within the channel affects the selectivity filter(s). If this latter case is in fact true, then more detailed analysis will clearly be necessary.
Figure 1A shows two data sets taken from Wang and Veenstra (20) for $\mathrm{Cx} 43$ where unitary conductance is plotted against the cation mobility of the major cation in the pipette solution when $\mathrm{Cl}$ is the major anion (circles). Unitary conductance was also plotted against anion mobility (triangles) where the major cation is $\mathrm{K}$. The solid lines represent linear regression fits of the data. The thicker of the two lines represents the anion data where the Y-intercept denotes the residual cation conductance of $41 \mathrm{pS}$. The thinner line represents the cation data where the Y-intercept is the residual anion conductance of $27 \mathrm{pS}$. Figure 1B represents the same analysis for $\mathrm{Cx} 40$. The circles represent cation data for $\mathrm{Cx} 40$ and the triangles anion data. The homotypic $\mathrm{Cx} 43$ seems to be a highly non-selective channel where ionic mobility is the rate limiting factor for permeation. For homotypic $\mathrm{Cx} 40$ the data imply an interaction between highly mobile anion species and cations which hampers cation transit through the channel. In fact, as the anion mobility declines the unitary conductance increases, indicating that some interaction between cation and anion in $\mathrm{Cx} 40$ channels could be occurring (23).

Data for Cx37 are shown in Figure 2 which reveals a potential hazard to the conventional interpretation for such data. Spe-

Figure 1 -A, Selectivity sequence of $\mathrm{C} \times 43$ for cations and anions taken from Wang and Veenstra (20). Unitary conductance is plotted against equivalent conductivity $\left(\mathrm{cm}^{2} / \mathrm{Ohm}\right)$. The triangles represent unitary conductances measured with different anions, with $\mathrm{K}^{+}$as the cation. The circles are unitary conductance measured with different cations, with $\mathrm{Cl}^{-}$as the anion. The lines represent the linear regression fits to the data points. The thick solid line is the fit for the anions while

the thinner line is for the cations. The y-intercept for the anions represents the conductance due to $\mathrm{K}^{+}(41 \mathrm{pS})$ while the y-intercept for the cation data represents the $\mathrm{Cl}^{-}$conductance (27 pS). B, Cx40 taken from Beblo and Veenstra (23). The analysis is the same as that for Cx43. With constant $\mathrm{K}^{+}$as the anion species, increases in size and decreases in mobility resulting in increased channel conductance imply a cation/anion interaction for the more mobile anions which reduces conductance. The y-intercepts again represent the cation conductance (171 pS) and anion conductance (8 pS), respectively. 
cifically, note that the y-intercept is $-49 \mathrm{pS}$, the theoretical $\mathrm{Cl}$ conductance. Such behavior predicts that the unitary conductance $v s$ cation/anion concentration will be non-linear in the $\mathrm{mM}$ range. This has been shown to be the case for $\mathrm{Cx} 37$ (29) and is a ready explanation for the aberrant $\mathrm{Y}$-intercept.

For some connexins the permselectivity properties might be dramatically altered by the ionic environment. Gap junction membrane conductance is known to be modulated by a number of molecules which also are able to diffuse through the gap junction channel (30). For example, hydrogen and calcium ions are two species which cause the channel to gate closed in sufficiently high concentration, but in turn both are able to diffuse through the channel at concentration ranges normally found in cells $(31,32)$. It could be that cation and anion species also affect gap junction channel gating as well as the permeation path. There is now evidence for the supposition that the concentration of monovalents affects the gating of $\mathrm{Cx} 43$ through a screening mechanism (29). But for Cx37 homotypic gap junction channels the monovalent cation species has no detectable effect. Rather, $\mathrm{Mg}^{2+}$ is effective in altering the kinetics of the voltage dependence (33). However, with respect to $\mathrm{Cx} 37$, there are still no data available on the screening effects and/or the binding of specific sites on the permeation path.

Another technical approach which has been utilized to document the poor selectivity characteristics of gap junctions is the transfer of fluorescent probes. Few attempts have been made to quantify transfer rates, but dye transfer has been demonstrated for the majority of the mammalian connexins. In one study the transfer of carboxyfluorescein and dichlorofluorescein was shown for $\mathrm{Cx} 45$, Cx43, Cx40 and Cx37 (18). Millimolar amounts of dye were introduced into one cell of a pair via a whole cell patch. After $10 \mathrm{~min}$, fluorescence was monitored. Cell pairs were scored either as having transferred probe to the adjacent cell or not. The junctional conductance was also determined. For all the connexin types studied, the minimal conductance for observing dye transfer was in the 2-5 $\mathrm{nS}$ range. These data indicate that many, if not all, connexins are capable of passing both cations and anions of some size and charge. At issue is not the poor selectivity but rather the permeation rates necessary to allow specific messenger molecules to activate/inhibit whole groups of cells. This latter point is very important to answer if we are going to be able to understand the role of gap junctions in non-excitable tissue rather than continue to have speculation as the sole basis of further investigation.

\section{Are heterotypic or heteromeric channel forms important to function?}

An interesting issue which has yet to be addressed with regard to permselectivity is whether or not heteromeric and/or heterotypic biophysical properties are sufficiently different from homotypic channels. Sufficiently different, that is, to affect the cell to cell diffusion of critical substances able to trigger/alter cellular responses. In the vascular wall this is of particular interest because of the connexin co-expression that occurs in endothelial and vascular smooth muscle cells.

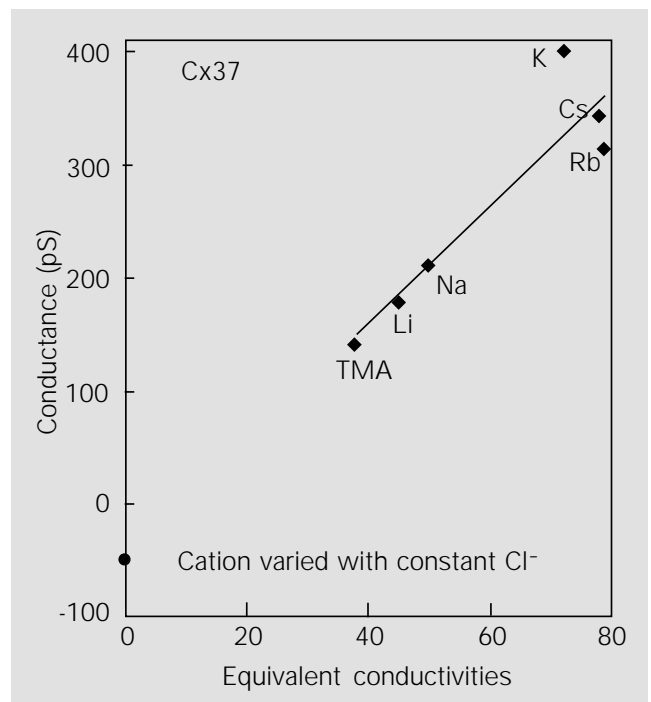

Figure 2 - Measured unitary conductance for $\mathrm{C} \times 37$ with various cations, with $\mathrm{Cl}^{-}$again remaining constant. The data indicate that the $\mathrm{Cl}^{-}$conductance is a negative number. This illustrates that the analytical approach used has limits. It implies that channel ion interactions could play a role in determining the nature of the permselectivity and that different ionic conditions will yield different permeation rates for the same ion. TMA, Tetramethylammonium. 
A question which has yet to be answered is the gap junction types which are present in co-expressing cells in situ. Heterotypic and heteromeric channel types have been demonstrated in vitro, implying that the same types of channels could form in situ $(14,34)$. In this regard, it becomes obvious that permselectivity measurements of the heterotypic, heteromeric and, to a lesser extent, homotypic channel types in situ are not going to be readily available in the near future.

Dye transfer between cells co-expressing connexins has been documented in situ (35) but quantitative analysis has not been forthcoming to allow comparison with cells expressing a single connexin. An interesting observation made by Little et al. (35) was the lack of dye transfer from smooth muscle cells of the tunica media to endothelial cells in the tunica intima. The reverse transfer (from endothelial to smooth muscle cells) was demonstrable. This type of result might well be best explained as one of the two cell types representing an infinite sink, the classic example of this phenomenon being that illustrated by Rae et al. (36).

In vitro studies of $\mathrm{Cx} 43$ and $\mathrm{Cx} 37$ have shown that $\mathrm{Cx} 43$ and $\mathrm{Cx} 37$ form heterotypic and heteromeric channels along with homotypic forms (14). The most notable parameter which was different in cells co-expressing $\mathrm{C} \times 34$ and $\mathrm{C} \times 37$ was a broadening of the $\mathrm{V}_{\mathrm{o}}$. The second feature was the occurrence of channel conductances that could not be explained as heterotypic or homotypic. The different conductive states imply, as indicated before, different permselectivity properties. This observation points to the issue already raised, i.e., that even if the permselectivity is different, is it different enough to affect tissue physiology? There is a second perplexing problem. In a given vascular tissue co-expressing distinct connexins, what population of channel types are there? What fraction of the total number of channels is homotypic, heterotypic or heteromeric? Without this information it is impossible to quan- tify the role that the three generic gap junction types might play in normal and abnormal tissue physiology.

\section{Conclusions and clinical relevance}

The major role played by intercellular communication through gap junctions in modulating vasomotor tone throughout the vascular tree has been discussed in detail elsewhere $(37,38)$. The implication for this report is that it follows naturally that since gap junctions play such a critical role in maintaining circulatory homeostasis, they are logical molecular targets for vascular disease, and as such, for vascular therapy. However, it should be emphasized that there are currently no published reports that have either confirmed or denied a direct proximal role for gap junctions in vascular disease.

More specifically, it is easy to imagine how increased intercellular coupling, for example, among vascular smooth muscle cells might contribute to the pathology of diseases that involve heightened contractility/impaired relaxation of the vascular smooth muscle cell (i.e., hypertension, Raynaud's disease, coronary artery disease, erectile dysfunction, etc.). In other words, an increase in intercellular coupling could lead to greater stimulus/impulse propagation among some population of coupled smooth muscle cells, thus leading to exaggerated contractile responses, and perhaps hypertension $(39,40)$. The opposite could be true for reduced endothelium-dependent relaxation responses. In addition, restenosis and atherosclerosis (41), disease states which result in significant remodeling of the vascular wall, might be expected to profoundly alter the degree of intercellular coupling and/or be affected by altered cell-to-cell communication among vascular wall cells. The implication of such changes to vascular function have hardly been investigated. Moreover, it should be emphasized that the role of endothelialsmooth muscle cell coupling in the regula- 
tion of vascular tone is just beginning to be studied. There are many possibilities, and it is clear the real work has just begun.

On the other hand, theoretical studies suggest that the degree of coupling among vascular smooth muscle cells may already be such that 10-fold or greater increases in coupling would be required for physiologically relevant changes in vascular contractility $(8,9)$. This is not to say that altered connexin expression would play no role in vascular pathology, but that intercellular communication is so critical to vascular function, that there is significant plasticity or reserve to prevent it from becoming the focal point for tissue pathology. Of course all of the latter considerations are based on the known biophysical characteristics of $\mathrm{Cx} 43$ in human vascular smooth muscle, i.e., long open times, high open probabilities, physiologically irrelevant subconductance states, and lack of selectivity. In this regard, however, if there are indeed physiological or pathophysiologi- cal alterations in second messenger molecules/ions that result in dramatic changes in the documented biophysical behaviors of connexins, then the role of gap junctions could be drastically altered. Dynamic ligand gating of substate dwell times or permselectivity properties, for example, or the presence of significant heteromeric or heterotypic channel types, could have profound implications for normal vascular physiology and disease. Certainly the potential clinical correlates of altered intercellular communication can be visualized, but have not yet been documented. On this basis, this would seem to be a very fruitful area of future research.

\section{Acknowledgments}

The authors would like to acknowledge Dr. S.V. Ramanan, SUNY at Stony Brook, NY, USA, who kindly donated the data for Figure 2.

\section{References}

1. Christ GJ , Spray DC, ElSabban M, Moore LK \& Brink PR (1996). Gap junctions in vascular tissues: Evaluating the role of intercellular communication in the modulation of vasomotor tone. Circulation Research, 79: 631-646.

2. Segal SS \& Kurjiaka DT (1995). Coordination of blood flow control in the resistance vasculature of skeletal muscle. Medicine and Science in Sports and Exercise, 27: 1158-1164.

3. Beach J M, McGahren ED \& Duling BR (1998). Capillaries and arterioles are electrically coupled in hamster cheek pouch. American J oumal of Physiology, 275 (Part 2): $\mathrm{H} 1489-\mathrm{H} 1496$.

4. Pacicca C, Schaad O \& Beny J (1996). Electrotonic propagation of kinin-induced, endothelium-dependent hyperpolarizations in pig coronary smooth muscles. J ournal of Vascular Research, 33: 380385.

5. Bevan J A \& Torok J (1970). Movement of norepinephrine through the media of rabbit aorta. Circulation Research, 27: 325331.
6. Hirst GDS \& Edwards FR (1989). Sympathetic neuroeffector transmission in arteries and arterioles. Physiological Reviews, 69: 546-604.

7. Bumstock $G$ (1970). Structure of smooth muscle and its innervation. In: Bulbring $E$, Brading AF, J ones AW \& Tomita T (Editors), Smooth Muscle. Williams \& Wilkins, Baltimore, MD, 1-69.

8. Christ GJ , Brink PR \& Ramanan SV (1994). Dynamic gap junctional communication: a delimiting model for tissue responses. Biophysical J oumal, 67: 1335-1344.

9. Ramanan SV, Brink PR \& Christ GJ (1998). Neuronal innervation, intracellular signal transduction and intercellular coupling: a model for syncytial tissue responses in the steady state. J ournal of Theoretical Biology, 193: 69-84.

10. Beyer EC (1993). Gap junctions. International Review of Cytology, 137: 1-37.

11. Brink PR (1998). Gap junctions in smooth muscle. Acta Physiologica Scandinavica, 164: 349-356.

12. Brink PR, Valunias V \& Christ GJ (2000). Homotypic, heterotypic and heteromeric gap junctions. In: Perrachia C (Editor), Gap J unctions. Academic Press, New York.

13. Brink PR, Ramanan SV \& Christ GJ (1996). Human connexin43 gap junction channel gating evidence for mode shifts and/or heterogeneity. American J ournal of Physiology, 271: C321-C331.

14. Brink PR, Cronin K, Banach K, Peterson E, Westphale EM, Seul KH, Ramanan SV \& Beyer EC (1997). Evidence for heteromeric gap junction channels formed from rat connexin43 and human connexin37. American J ournal of Physiology, 273: C1386-C1396.

15. Valiunas $V$, Bukaukas $F \&$ Weingart $R$ (1997). Conductances and selective permeability of connexin43 gap junction channels examined in neonatal rat heart cells. Cirulation Research, 80: 708-719.

16. Moreno AP, Liang J G, Beyer EC \& Spray DC (1995). Properties of gap junction channels formed of connexin 45 endogenously expressed in human hepatoma (SKHep1) cells. American J ournal of Physiology, 268: C356-C365.

17. Moreno AP, Campos de Carvalho AC 
Christ GJ \& Spray DC (1993). Gap junctional communication between human corpus cavemosum smooth muscle cells in culture: Gating behavior and single channel events. American J ournal of Physiology, 264: C80-C92.

18. Campos de Carvalho AC, Moreno AP, Christ GJ , Melman A, Roy C, Hertzberg EL \& Spray DC (1993). Gap junctions formed of connexin43 interconnect smooth muscle cells of the human corpus cavernosum. J ournal of Urology, 149: 1568-1575.

19. Veenstra RD, Wang HZ, Beblo DA, Chilton MG, Harris AL, Beyer EC \& Brink PR (1995). Selectivity of connexin-specific gap junctions does not correlate with channel conductance. Circulation Research, 77: 1156-1165.

20. Wang H-Z \& Veenstra RD (1997). Monovalent ion selectivity sequences of the rat connexin 43 gap junction channel. J ournal of General Physiology, 109: 491-507.

21. Segal SS, Welsh DG \& Kurjiaka DT (1999). Spread of vasodilation and vasoconstriction along feed arteries and arterioles of hamster skeletal muscle. J oumal of Physiology, 516: 283-291.

22. Veenstra RD, Wang $H Z$, Beyer EC, Ramanan SV \& Brink PR (1994). Connexin37 forms high conductance gap junction channels with subconductance state activity and selective dye and ionic permeabilities. Biophysical J ournal, 66: 19151928.

23. Beblo DA \& Veenstra RD (1997). Monovalent cation permeation through the connexin 40 gap junction channel. J ournal of General Physiology, 109: 509-522.

24. Christ GJ \& Brink PR (1999). An analysis of the presence and physiological relevance of subconducting states of connexin43-derived gap junction channels in cultured human corporal vascular smooth muscle cells. Circulation Research, 84: 797-803.

25. Eisenmann G\& Horn R (1983). Ionic selectivity revisited: the role of kinetic and equilibrium processes in ion permeation through channels. J ournal of Membrane Biology, 331: 599-635.

26. Benz R (1986). Analysis and chemical modification of bacterial porins. In: Miller C (Editor), Ion Channel Reconstitution. Plenum Publishing Corp., New York.

27. Benz R, Kanko K \& Langer P (1980). Pore formation by the matrix protein (porin) of Escherichia coli in planar bilayer membranes. Annals of the New York Academy of Sciences, 258: 13-24.

28. Brink PR \& Fan SF (1989). Patch clamp recordings form membranes which contain gap junction channels. Biophysical J ournal, 56: 579-593.

29. Banach K, Ramanan SV \& Brink PR (1998). Homotypic hCx37 and rCx43 and their heterotypic form. In: Werner R (Editor), Gap J unction. IOS Press, Amsterdam, 7680.

30. Burt J \& Spray DC (1988). Inotropic agents modulate gap junctional conductance between cardiac myocytes. American J ournal of Physiology, 254: H1206-H1210.

31. Spray DC, Harris AL \& Bennett MVL (1981). Equilibrium properties of a voltage-dependent junctional conductance. J oumal of General Physiology, 77: 77-93.

32. Loewenstein W (1981). J unctional intercellular communication: The cell to cell membrane channel. Physiological Reviews, 61: 829-913.

33. Ramanan SV, Brink PR, Varadaraj $K$, Peterson E, Schirmacher K \& Banach K (1999). A three-state model for connexin37 gating kinetics. Biophysical J ournal, 76: 2520-2529.

34. Stauffer KA (1995). The gap junction proteins B1-connexin and B2-connexin can form heteromeric hemichannels. J ournal of Biological Chemistry, 270: 6768-6772.

35. Little TL, Xia J \& Duling BR (1995). Dye tracer defines differential endothelial and smooth muscle coupling pattems within the arteriolar wall. Circulation Research, 76: 498-504.

36. Rae J, Bartling C, Rae J \& Mathias RT (1996). Dye transfer between cells of the lens. J oumal of Membrane Biology, 150: 89-103.

37. Christ GJ, Brink PR, Xhao W, Moss J, Gondra J , Roy C \& Spray DC (1993). Gap junctions modulate tissue contractility and $\alpha_{1}$-adrenergic agonist efficacy in isolated rat aorta. J ournal of Pharmacology and Experimental Therapeutics, 266: 1054 1065

38. Christ GJ (1995). Modulation of $\alpha_{1}$-adrenergic contractility in isolated vascular tissues by heptanol: a functional demonstration of the potential importance of intercellular communication to vascular response generation. Life Sciences, 56: 709-721.

39. Watts SW, Tsai ML, Loch-Caruso R \& Webb RC (1994). Gap junctional communication and vascular smooth muscle reactivity: use of tetraethylammonium chloride. J ournal of Vascular Research, 31: 307-313.

40. Watts SW \& Webb RC (1996). Vascular gap junctional communication is increased in mineralocorticoid-salt hypertension (see comments). Hypertension, 28: 888-893.

41. Polacek D, Bech F, McKinsey J F \& Davies PF (1997). Connexin43 gene expression in the rabbit arterial wall: effects of hypercholesterolemia, balloon injury and their combination. J ournal of Vascular Research, 34: 19-30. 